\title{
The Microwave Sources for EPR Spectroscopy
}

\author{
Mariusz Hruszowiec, Kacper Nowak, Bogusław Szlachetko, Michał Grzelczak, \\ Wojciech Czarczyński, Edward F. Pliński, and Tadeusz Więckowski \\ Terahertz, Technology Center, Wrocław University of Science and Technology, Wrocław, Poland
}

\begin{abstract}
Rapid development of many scientific and technical disciplines, especially in material science and material engineering increases a demand for quick, accurate and cheap techniques of materials investigations. The EPR spectroscopy meets these requirements and it is used in many fields of science including biology, chemistry and physics. For proper work, the EPR spectrometer needs a microwave source, which are reviewed in this paper. Vacuum tubes as well as semiconductor generators are presented such as magnetron, klystron, traveling wave tube, backward wave oscillator, orotron, gyrotron, Gunn and IMPATT diodes. In this paper main advantages of gyrotron usage, such as stability and an increased spectral resolution in application to EPR spectroscopy is discussed. The most promising and reliable microwave source is suggested.
\end{abstract}

Keywords-electron paramagnetic resonance, gyrotron EPR, microwave sources, orotron, terahertz waves.

\section{Introduction}

Electron paramagnetic resonance (EPR) is a very sensitive and specialized method that can be applied for both element and chemical reaction investigations. The EPR method can be used to detect the organic and inorganic compounds in electrochemical systems. A form of the equation, describing an EPR resonance condition:

$$
h v=g \beta B,
$$

shows that an EPR signal can be observed in two ways.

The first is an observation of the resonant energy absorption versus frequency $v$ of the electromagnetic field at a constant magnetic induction $B$. The second way is to observe the resonant energy absorption versus magnetic induction $B$ of the constant magnetic field at a constant frequency $v$ ( $g$ is Lande factor).

Experiments using EPR are mainly conducted in X and Q bands, and less frequently in $\mathrm{V}$ and $\mathrm{W}$ ones. The common use of the $\mathrm{X}$ and $\mathrm{Q}$ bands is due to their wide availability and the relatively low price of all microwave components (developed for radar systems). The second reason for the wide use of the $\mathrm{X}$ and $\mathrm{Q}$ bands is the use of comparatively weak constant magnetic fields with an induction of the order of $1 \mathrm{~T}$, which is achievable with conventional electromagnets. Development of such electromagnets is relatively simple and cheap. Their operation is much more convenient than superconductive electromagnets, for instance. However, a spectroscopic splitting factor for these bands limits the ability to examine the substances, which exhibit low magnetic anisotropy.

Measurements carried out in the millimeter wave band in a frequency range above $40 \mathrm{GHz}$ introduce a high resolution of a spectrum for a given $g$ coefficient. Spectral resolution increases with radiation frequency and the intensity of the constant, resonant magnetic $B_{0}$. It has been demonstrated in past work [1] based on analysis of different biological, polymeric and other spin systems examined in the D band. There may be several criteria for selecting a millimeter wave source for EPR, including performance, output power, tuning bandwidth, durability, convenience and costs [2]. Solid-state sources such as Gunn and IMPATT diodes are often chosen because of their relatively low cost and compact dimensions. Due to them having small dimensions, it is easier to integrate them into EPR devices. What is more, solid-state sources do not require high control voltages and additional cooling. However, they can only deliver low output power compared to vacuum tubes.

Table 1

Example bands used in EPR spectroscopy

\begin{tabular}{|l|c|c|c|c|c|c|c|}
\hline Band & L & X & K & Q & V & W & D \\
\hline$\lambda[\mathrm{mm}]$ & 300 & 30 & 12.5 & 8.5 & 5.6 & 3.2 & 2.1 \\
\hline$f[\mathrm{GHz}]$ & 1 & 10 & 24 & 35 & 65 & 95 & 140 \\
\hline$B_{0}[\mathrm{~T}]$ & 0.04 & 0.36 & 0.86 & 1.25 & 2.3 & 3.39 & 5.00 \\
\hline
\end{tabular}

\section{Sources of the Microwave Radiation}

\subsection{Magnetron}

Magnetrons were the first microwave devices used for the generation of high frequency radiation. The first magnetron was constructed by Albert Hull in 1920. A modern project of the magnetron device was proposed during the Second World War in 1940 by John and Harry Boot from the University of Birmingham. Engineers managed to construct radars, which despite their pulsed operation contributed to the victory of the Allies over Germany. Nowadays, most magnetrons are used in households, where they work as 
a source of the microwaves in microwave ovens, whereas in radar applications the most frequently used sources are klystrons and backward-wave tubes.

The magnetron can generate high power radiation [3] (up to hundreds of kilowatts) and high frequency, which is limited mainly by the intensity of a constant magnetic field according to the formula

$$
f=\frac{e B}{m c}
$$

where $f$ denotes the frequency of the electron oscillations, $e$ is the charge of the electron, $B$ is the induction of an external constant magnetic field, $m$ is the mass of the electron and $c$ is the speed of light. The magnetron is characterized by a high instability of both a generated frequency and its phase [4]. Due to these disadvantages, magnetrons are not used in EPR spectroscopy.

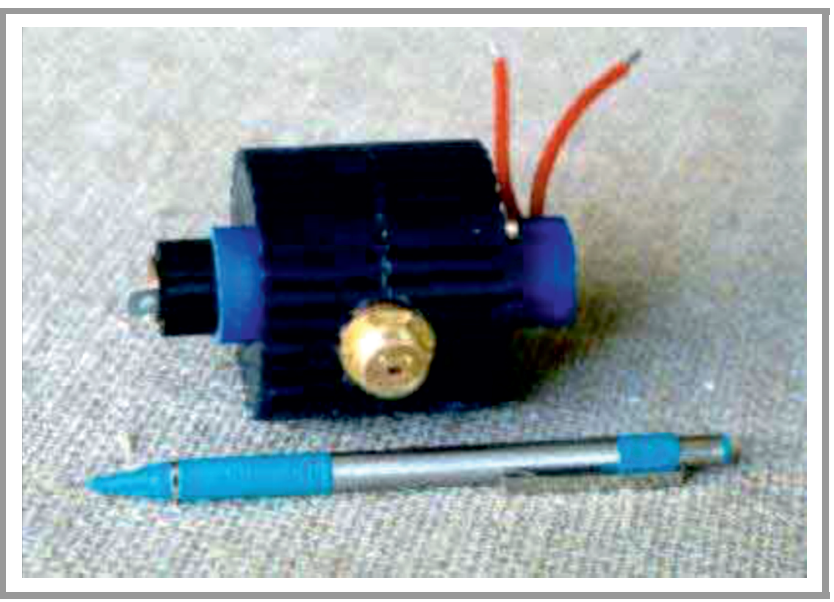

Fig. 1. The SHM magnetron delivers $1 \mathrm{~kW}$ output power at $95 \mathrm{GHz}$ [8], [10].

Nevertheless, the constant progress in magnetron development for higher frequencies and output powers can be noticed. In the most high frequency magnetrons a new type of this device is used, the so-called spatial-harmonic magnetron (SHM), which utilizes a cold secondary-emission cathode. It was first proposed by Robertshaw [5] and lately reintroduced by [6], along with the whole theory for multimode interactions, formation of doublets, and transient processes. Based on this theory several devices were designed and built for $95 \mathrm{GHz}$ (Fig. 1) [7] and $210 \mathrm{GHz}$ [8]. Another magnetron for higher frequency $(320 \mathrm{GHz})$ is under development [9]. The SHM magnetrons could open a new age of magnetrons and despite their disadvantages they have very important advantages such as their small size and low price in comparison with other microwave tubes, e.g. klystrons and gyrotrons.

\subsection{Gunn Diode}

Historically, one of the first semiconductor sources of microwave radiation was a Gunn diode [11]. It was used as an active element in many microwave generators and amplifiers. Thanks to its simple construction and an ability to generate oscillations at very high frequencies, it is used in many systems. Diodes based on gallium arsenide are able to generate radiation at a frequency reaching $200 \mathrm{GHz}$, while frequency reachable by the diodes based on gallium nitride can be as high as $3 \mathrm{THz}$ [12]. Moreover, Gunn generators are tunable in a wide frequency range [13]. Unfortunately, the achieved power is low and does not exceed several milliwatts CW. However, this power level is sufficient to be used in EPR spectroscopy.

As was written in [2], Gunn diodes can provide up to $100 \mathrm{~mW} \mathrm{CW}$ radiation at $95 \mathrm{GHz}$ and $20 \mathrm{~mW}$ at $140 \mathrm{GHz}$. The main disadvantage of solid-state sources is poor noise and frequency jitter characteristics, which must usually be compensated by phase-lock loops to a reference oscillation source or external cavity. For example, the Gunn diode working at $95 \mathrm{GHz}$ has a phase noise of about $-40 \mathrm{dBc} / \mathrm{Hz}$ at a $10 \mathrm{kHz}$ frequency offset. However, phase locking limits the sweep ability of the necessary microwave sources e.g. for control matching the EPR cavity.

The diode based oscillators can be expected to work continuously for up to $100,000 \mathrm{~h}$, which is a much longer working time than vacuum tubes could offer.

\subsection{Klystron}

Klystrons, as well as magnetrons, were invented at the beginning of the XX century. The main work was done by the Varian brothers [14]. Klystrons can work as amplifiers as well as microwave generators for a wide range of wavelengths (from radio frequencies up to upper microwaves). Klystrons are widely used in such areas as radars, TV transmitters, satellite communication and in modern particle accelerators [15].

A principle of klystron operation is based on a conversion of the energy of accelerated electrons to the energy of the electromagnetic field. This conversion occurs due to the bunching of electrons in the alternating electric field. A klystron usually consists of an electron gun, a resonance cavity (or several cavities) and a collector or reflector. In a typical generation klystron, which is the reflex klystron [16].

Klystrons are stable sources of electromagnetic radiation, which can be successfully used in EPR spectroscopy [17]. In a typical EPR instrument, a klystron tube is used to generate monochromatic microwave radiation in the $\mathrm{X}$ band $(\approx 9.75 \mathrm{GHz})$, but all other bands are also used.

Although they also have limits. The main limit for klystrons is the highest frequency, which can be generated at a reasonable power level (tens of watts). The ratio between the generated frequency and the output power for modern klystrons drops rapidly when working frequency reaches $100 \mathrm{GHz}$ and above [18] (Fig. 2).

One solution to the problem of output power loss generated by klystrons was the development of the so-called 


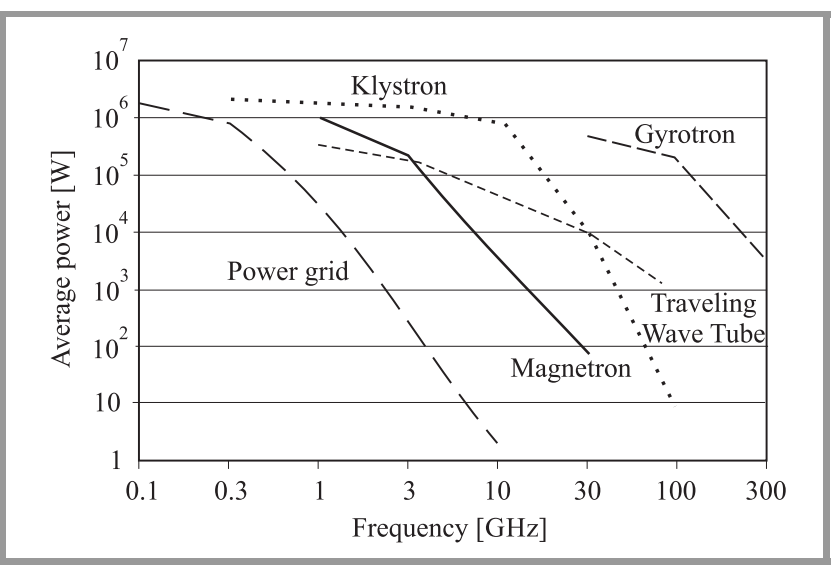

Fig. 2. Diagram presenting power versus frequency limits for microwave generators [18].

extended interaction klystrons (EIK), which combine the advantages of normal klystrons and those of traveling wave tubes (TWT) [19]. It is said that EIK klystrons will reach very high frequencies (up to $1 \mathrm{THz}$ ), and at the same time with a high power level and frequency stability. In the present day, there is research on klystrons that will work on a frequency of $670 \mathrm{GHz}$ [20].

\subsection{Traveling Wave Tube}

As with the previously mentioned radiation sources, the travelling wave tube (TWT) was also invented during the Second World War [21]. It is said that the creators of these kinds of tubes are Rudolf Kompfner and Nils Lindenbland. The principle of operation is similar to klystrons, which is also based on extraction of energy from accelerated electrons and the transfer of part of it to the electromagnetic field. However, in this case the delaying structure is used, which causes electrons to move with a velocity close to the alternation of the electromagnetic field. Electrons are periodically focused by magnets or electromagnets, which, as mentioned before, cause the occurrence of the bunching of electrons.

TWTs are usually used as amplifiers of microwave radiation and they are characterized by a wide bandwidth, which make TWTs very good broadband amplifiers. In contrast to klystrons, TWTs are able to reach much higher output powers at frequencies above $100 \mathrm{GHz}$. Due to this fact, TWTs are broadly used in many areas such as, among others, telecommunications, radar techniques and EPR [22].

\subsection{Backward Wave Oscillator (BWO)}

A backward wave oscillator (BWO), also called a backward wave tube, is a vacuum tube that was invented by the inventor of the traveling wave tube, Rudolf Kompfner [21]. He demonstrated O-type BWO in 1951, whereas M-type was presented by Bernard Epsztein [23] in the same year. The BWO is used to generate microwaves up to the terahertz range. It also belongs to the traveling-wave tube (TWT) family and is an oscillator, which can be tuned in a wide electronic range.

The principle of operation of the BWO is based on the interaction between an electron beam, which is generated by an electron gun, and a slow-wave structure. The excited microwave wave is traveling backward against the beam. When it reaches the electron gun region, it is coupled out by the output port. The BWOs are considered as very stable and powerful microwave sources, although the maximum output power level for O-type is in a range up to $1 \mathrm{~mW}$ at $1000 \mathrm{GHz}$. Nevertheless, the good quality wavefront they produce makes them very good illuminators in terahertz imaging and EPR spectroscopy. The BWOs are widely used in EPR spectroscopy, for example a set of four BWOs were used in [24] in so called high frequency EPR (HFEPR), where high fields are employed in combination with multiple energy sources in the sub-THz range.

Another example of using the BWO as microwave sources can be found in [25], where results of studying the structure of the paramagnetic centres formed by impurity $\mathrm{Ho}^{3+}$ ions in synthetic forsterite $\left(\mathrm{Mg}_{2} \mathrm{SiO}_{4}\right)$ are presented. They used a basic set of BWOs which permit operation in a frequency range of 60 to $1500 \mathrm{GHz}$ [26]. Unfortunately, the output power of the BWO at frequencies over $100 \mathrm{GHz}$ are at miliwatt level. This factor limits usage of the BWOs at higher frequencies and one needs to use other microwave sources.

\subsection{Orotron}

The orotron was proposed in 1969 by the Soviet physicists F. S. Rusin and G. D. Bogomlov [27]. It consists of an open resonator (Fig. 3) with two mirrors, one of which has a periodic structure. The electron beam is located in a focusing magnetic field directed parallel to the motion of the electrons. The periodic structure produces the slow spatial harmonics of the cavity field. Electrons interact with one of such harmonics under the condition:

$$
\omega \approx h v
$$

and then a simple relation between the radiation wavelength $\lambda$ and structure period $d$ can be used:

$$
\lambda \approx \frac{d}{\beta}
$$

where $\omega$ is the angular frequency of the cavity mode, $h=\frac{2 \pi}{d}$ is the longitudinal wavenumber of the slow spatial harmonic of the field, $v$ is the electron velocity, $\beta=\frac{v}{c}$ and $c$ is the speed of light.

Orotrons are currently being developed for generating high frequency radiation [28], [29] of up to $410 \mathrm{GHz}$ at a 50-200 $\mathrm{mW}$ power level. Nevertheless, the efficiency of this device is quite low due to the large number of periods in the periodic structure and the non-uniformity of the microwave field magnitude at the electron beam cross section. 

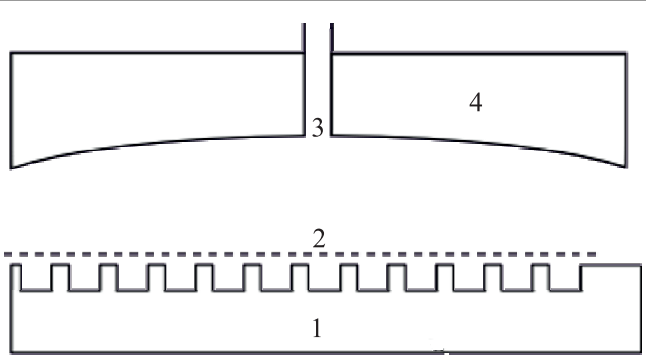

Fig. 3. Structure of an orotron: 1 - mirror with periodic structure, 2 - electron flux, 3 - radio wave guide, 4 - mirror.

Efficiency does not usually exceed $0.1-1 \%$ at submililimeter wavelengths [30].

In contrast to the gyrotron, the orotron is a smaller and more compact device. It also does not require a high intensity of the magnetic field. What is more, it does not require a complicated power supply and cooling systems. Therefore, the output power levels generated by the orotron are several orders of magnitude less than those generated by the gyrotron.

\subsection{IMPATT Diode}

The IMPATT diode (IMPact ionization Avalanche TransitTime diode) is a powerful semiconductor device used to produce millimetre waves and microwaves. Its structure, similar to the basic PN junction, was first proposed by Shockley (1954) [31] and further developed by Read (1958) [32].

Transit time of the carriers in the junction results in the negative resistance effect, which is responsible for the generation of the RF signal. IMPATT diodes cover the frequency range from X-band $(6 \mathrm{GHz})$ to $400 \mathrm{GHz}$. Their $\mathrm{CW}$ power was reported at $12 \mathrm{~W}$ at $6 \mathrm{GHz}, 1 \mathrm{~W}$ at 94 $\mathrm{GHz}$ [33] and $2.2 \mathrm{~mW}$ at $412 \mathrm{GHz}$ [34].

These kind of generators are widely used in EPR spectroscopy, especially in the former Soviet Union where this technology was highly developed [35].

The main advantage of IMPATT diodes is their noiseperformance, which is comparable to klystrons. What is more, the price is much lower, the lifetime is long and only a simple power supply is required [36]. The only drawback of the IMPATT diodes is a high level of phase noise, which is not desirable at EPR spectrometer setup. Considering all disadvantages and advantages the IMPATT diodes can be very promising microwave source, but one need to use proper methods to compensate the phase noise.

\subsection{Gyrotron}

The devices that are widely used as a microwave source in EPR/NMR experiments are gyrotrons [37], [38]. The gyrotron was invented in the Radiophysics Institute in Gorky (now the Applied Physics Institute in Nizhny Novogorod) in the 1960s [39]. The gyrotron is a kind of maser (strictly speaking it is a cyclotron resonance maser-CRM), which is equivalent to a laser for microwave wavelength, and as with the laser, the gyrotron is also an oscillator. This device is used with success in many experiments, where a stable, high frequency and high power microwave source is needed. The highest frequency reached by the gyrotron is now about $680 \mathrm{GHz}$ [40], with an output power level reaching about $300 \mathrm{~kW}$.

Despite the main application for gyrotrons being plasma heating in tokamak installations, they have attracted the attention of many researchers that are conducting experiments involving various kinds of spectroscope. For the last few years NMR/DNP [41] spectroscopy experiments have been widely reported. The first commercial NMR/DNP spectroscopes are available. There is continuous research towards higher radiation frequencies, which was recently reported during a workshop at University of Fukui, a leading centre in gyrotron research [42].

During the above-mentioned workshop in Fukui, the latest research results in the field of ESR spectroscopy, which involved the use of the gyrotron, were presented [43]. The experiments were conducted with the use of the gyrotron, which operated at a frequency of $154 \mathrm{GHz}$ and with an output power of $150 \mathrm{~W}$. The use of this kind of system will allow for further development of material engineering, especially in the context of materials with a short relaxation time. Similar research is being held in many other institutions e.g. in MIT [14], where the gyrotron for use in NMR spectroscopy was designed and built. It operates at a frequency of $460 \mathrm{GHz}$ with a maximal output power of $100 \mathrm{~W}$.

The gyrotron, as an electromagnetic radiation source, is a very promising device, mainly due to its simple construction, high efficiency and potential possibility to achieve frequencies of up to several terahertzes. The development of the gyrotron technique and technology is in the scope of interest of several countries, including Poland [44].

Frequency and power stability in the gyrotron has been studied for some time. MIT has been conducting experiments with a tunable gyrotron for spectroscopy applications since 1990 [45]. There is worldwide interest in this type of device. The gyrotron reported in [45] has a long term power stability of $0.7 \%$ at $2.71 \mathrm{~W}$ of power and $\pm 6 \mathrm{ppm}$ frequency stability at $460 \mathrm{GHz}$. On the other hand, the gyrotron reported in 2002 [41] achieved a power stability of $1 \%$. It is quite an improvement as the gyrotron reported in 1994 was operating at a frequency of $95 \mathrm{GHz}$ with a frequency stability of $100 \mathrm{ppm}$ [46]. The same group from Fukui, Japan reported in 2010 a wide range $(1.5 \mathrm{GHz})$ continuous tunable gyrotron working at $394.6 \pm 0.6 \mathrm{GHz}$ [37]. Unfortunately in this work, no stability test results nor measurement sensitivity gain have been reported.

\section{Summary}

The most important microwave sources were briefly discussed and the need of new microwave sources and the development of existing ones has been pointed out. 
Before some advice will be given on choosing the proper microwave oscillator for use in EPR, let once more time revise the reason why one need to use higher frequency.

There are several advantages of a higher frequency source in EPR applications [24]:

1. Second-order effects are reduced at high magnetic fields. Higher radiation frequency allows the use of the higher magnetic field.

2. A higher magnetic field and higher operation frequency increases spin splitting, thus spectral resolution over the $g$ factor is higher. Better resolution is important in the investigation of polarity, structure and spin.

3. Exponential dependency of the number of excited spins on the radiation frequency causes saturation of paramagnetic centers. This dependency is used to study the relaxation and dynamics of the paramagnetic centers. Moreover, cross-relaxation of the paramagnetic centers decreases rapidly at high magnetic fields. As a result it is possible to obtain more precise and complete information about the system under study.

4. Large microwave quantum energy makes it possible to investigate systems with large zero field splitting.

5. Precision of pulse methods also increases at high magnetic fields.

6. Higher orientation selectivity and sensitivity in the investigation of disordered systems.

The choice of the microwave source for the given application must meet all requirements and other specific criteria. Every oscillator has its advantages and disadvantages, and this must be carefully considered.

The most important among them, in the context of improving sensitivity and also measurement dynamics and the further development of spectroscopy, are the gyrotron and orotron.

The gyrotron, because of its high efficiency (up to 50\%) and also its high power and ability to generate a continuous wave of high frequency, will allow samples during a long exposure time in constant radiation conditions to be investigate.

But the gyrotron is very expensive and troublesome device and even though it has many advantages, in experiments required frequencies up to $100 \mathrm{GHz}$ can be used other microwave source. The gyrotron is the only choice when one need to operate at higher frequencies, but then much more powerful magnetic field is also needed.

The orotron, because of its compact size and also its pulsed operation and lack of complicated gear (power supply, magnets, cooling system), will allow samples to be even quickly examined in field conditions.

Orotrons can be used as an alternative to the other microwave tubes as well as semiconductor diodes. Orotron can provide higher power levels than diodes and other tubes, so it can be used as the intermediate source between oscillators of lower frequencies with higher output power and those with higher frequencies and high output power, i.e. gyrotron.

When one need to operate at low frequency the proper choice is gun/IMPATT diodes and cheap vacuum tubes such as TWTs, magnetrons and klystrons.

\section{Acknowledgements}

We acknowledge Andrzej Francik for very fruitful discussions.

\section{References}

[1] V. I. Krinichnyi, 2-mm Wave Band EPR Spectroscopy of Condensed Systems. Taylor \& Francis, 1994.

[2] K. Möbius and A. Savitsky, High-Field EPR Spectroscopy on Proteins and their Model Systems: Characterization of Transient Paramagnetic States. RSC Publishing, 2008

(doi: 10.1039/9781847559272).

[3] W. Willshaw, L. Rushforth, A. Stainsby, R. Latham, A. Balls, and A. King, "The high-power pulsed magnetron: development and design for radar applications", J. of the Institution of Elec. Engin. Part IIIA: Radiolocation, vol. 93, no. 5, pp. 985-1005, 1946 (doi: 10.1049/ji-3a-1.1946.0188).

[4] M. Neubauer, R. Johnson, A. Moretti, and M. Popovic, "Phase and frequency locked magnetrons for SRF sources", in Proc. Particle Accelerator Conference PAC09, Vancouver, Canada, 2009 [Online]. Available: http://lss.fnal.gov/archive/2009/conf/fermilab-conf09-202-ad.pdf

[5] R. Robertshaw and W. Willshaw, "Some properties of magnetrons using spatial-harmonic operation", Proceedings of the IEE - Part C: Monographs, vol. 103, no. 4, pp. 297-306, 1956 (doi: 10.1049/pi-c.1956.0041).

[6] S. Sosnytskiy and D. Vavriv, "Theory of the spatialharmonic magnetron: an equivalent network approach", IEEE Trans. on Plasma Sci., vol. 30, no. 3, pp. 984-991, 2002 (doi: 10.1109/TPS.2002.801616)

[7] J.-I. Kim, S.-G. Jeon, G.-J. Kim, J. Kim, V. D. Yeryomka, A. S. Tishchenko, and V. D. Naumenko, "Numerical and experimental investigation of a $35 \mathrm{GHz} 20$-vane spatial-harmonic magnetron", in Proc. 35th Int. Conf. on Infrared Millimeter and Terahertz Waves IRMMW-THz 2010, Rome, Italy (doi: 10.1109/ICIMW.2010.5613045).

[8] N. Avtomonov, V. Naumenko, D. Vavriv, K. Schunemann, A. Suvorov, and V. Markov, "Toward terahertz magnetrons: 210-GHz spatial-harmonic magnetron with cold cathode", IEEE Trans. on Elec. Dev., vol. 59, no. 12, pp. 3608-3611, 2012 (doi: 10.1109/TED.2012.2217974).

[9] N. Avtomonov, V. Naumenko, and D. Vavriv, "Development of terahertz spatial-harmonic magnetrons", in Proc. Eur. Microw. Conf. EuMC 2013, Nuremberg, Germany, 2013, pp. 187-190.

[10] "Spatial-harmonic magnetrons with cold secondary emission cathode" [Online]. Available: http://radar.kharkov.com/index.php?s= $3 \& \mathrm{p}=7$ (accessed Jan. 1, 2016).

[11] J. Gunn, "Microwave oscillations of current in III-V semiconductors", Solid State Commun., vol. 1, no. 4, pp. 88-91, 1963 (doi: doi.org/10.1016/0038-1098(63)90041-3) [Online]. Available: http://www.sciencedirect.com/science/article/pii/0038109863900413

[12] Z. Gribnikov, R. Bashirov, and V. Mitin, "Negative effective mass mechanism of negative differential drift velocity and terahertz generation", IEEE J. of Selec. Topics in Quantum Electron., vol. 7, no. 4, pp. 630-640, 2001, (doi: 10.1109/2944.974235).

[13] J. Carlstrom, R. Plambeck, and D. Thornton, "A continuously tunable 65 - 15-GHz Gunn oscillator", IEEE Trans. on Microw. Theory and Techni., vol. 33, no. 7, pp. 610-619, 1985 (doi: 10.1109/TMTT.1985.1133036). 
[14] M. Hornstein, R. Griffin, J. Machuzak, M. Shapiro, R. Temkin, and K. Kreischer, "A $460 \mathrm{GHz}$ gyrotron oscillator for use in DNP/NMR spectroscopy", in IEEE Conference Record - Abstracts Pulsed Power Plasma Science, 2001, Las Vegas, NV, USA, 2001, p. 516 (doi: 10.1109/PPPS.2001.961319).

[15] R. Stringall and J. Lebacqz, "High-power klystron development at the Stanford Linear Accelerator Center", in Int. Electron Devices Meeting 1970, Washington D.C., 1970, vol. 16, p. 128 (doi: 10.1109/IEDM.1970.188317).

[16] G. Caryotakis, "High power klystrons: Theory and practice at the Stanford Linear Accelerator Center. Part 1. Theory and design", SLAC-PUB 10620, United States Department of Energy Office of Science, 2004.

[17] H. A. Buckmaster and J. C. Dering, "A 9-GHz, single klystron, EPR spectrometer using superheterodyne demodulation", Canadian J. of Phys., vol. 45, no. 1, pp. 107-117, 1967 (doi: 10.1139/p67-012).

[18] "Microwave Processing of Materials", National Materials Advisory Board Commission on Engineering and Technical Systems, National Research Council (doi: 10.17226/2266).

[19] A. Roitman, R. Dobbs, D. Berry, M. Hyttinen, P. Horoyski, and B. Steer, "Advantages of the extended interaction klystron technology at millimeter and submillimeter frequencies", in IEEE 34th Int. Conf. on Plasma Sci. ICOPS 2007, Albuquerque, NM, USA, 2007 (doi: 10.1109/PPPS.2007.4345972).

[20] R. Dobbs et al., "Fabrication and test of terahertz extended interaction klystrons", in Proc. 36th Int. Conf. on Infrared Millimeter and Terahertz Waves IRMMW-THz 2011, Houston, TX, USA, 2011 (doi: 10.1109/irmmw-THz.2011.6105058).

[21] R. Kompfner, "The invention of traveling wave tubes", IEEE Trans. on Electron Dev., vol. 23, no. 7, pp. 730-738, 1976 (doi: 10.1109/T-ED.1976.18477).

[22] S. Fernandez-Gutierrez, D. Gautreau, J. Sirigiri, B. Popovic, D. Gamzina, and N. Luhmann, " $263 \mathrm{GHz}$ traveling wave tube (TWT) amplifier for dynamic nuclear polarization (DNP) and electron paramagnetic resonance (EPR) spectroscopy", in Proc. 40th Int. Conf. on Infrared Millimeter and Terahertz Waves IRMMW-THz 2015, Hong Kong (doi: 10.1109/IRMMW-THz.2015.7327797).

[23] B. Epsztein, "Backward flow travelling wave devices", US Patent 2,932,760, Apr. 1960 [Online]. Available: http://www.google.com/patents/US2932760

[24] J. Telser et al., "High-frequency/high-field EPR spectroscopy of the high-spin ferrous ion in hexaaqua complexes", Magnetic Resonance in Chemistry, vol. 43, no. S1, pp. S130-S139, 2005 (doi: $10.1002 / \mathrm{mrc}$.1689).

[25] A. Konovalov and V. Tarasov, "Millimeter and submillimeter EPR spectroscopy", Radiophys. and Quant. Electron., vol. 50, no. 10-11, pp. 813-822, 2007 (doi: 10.1007/s11141-007-0072-2).

[26] J. Krzystek, S. Zvyagin, A. Ozarowski, S. Trofimenko, and J. Telser, "Tunable-frequency high-field electron paramagnetic resonance", J. of Magnetic Resonance, vol. 178, no. 2, pp. 174-183, 2006 (doi: 10.1016/j.jmr.2005.09.007) [Online]. Available: http://www.sciencedirect.com/science/article/pii/ S1090780705003095

[27] F. Rusin and G. Bogomolov, "Orotron - an electronic oscillator with an open resonator and reflecting grating", Proceedings of the IEEE, vol. 57, no. 4, pp. 720-722, 1969 (doi: 10.1109/PROC.1969.7049).

[28] A. Fedotov, V. Bratman, B. Dumesh, P. Makhalov, and F. Rusin, "Orotron oscillators and frequency multipliers as sources of coherent terahertz radiation", in Proc. Int. Worksh. Terahertz and Mid Infrared Radiation: Basic Res. and Pract. Appl. TERA-MIR 2009, TurunçMarmaris, Turkey, 2009, pp. 19-20 (doi: 10.1109/TERAMIR.2009.5379648).

[29] V. Bratman, B. Dumesh, A. Fedotov, P. Makhalov, B. Movshevich, and F. Rusin, "Terahertz orotrons and oromultipliers", IEEE Trans. on Plasma Sci., vol. 38, no. 6, pp. 1466-1471, 2010 (doi: 10.1109/TPS.2010.2041367).

[30] G. S. Nusinovich, "Analytical nonlinear theory of the orotron", Phys. of Plasmas, vol. 13, no. 5, 2006 (doi: 10.1063/1.2200631).

[31] W. Shockley, "Negative resistance arising from transit time in semiconductor diodes", Bell System Tech. J., vol. 33, no. 4, pp. 799-826, 1954.
[32] W. Read, "A proposed high-frequency, negative resistance diode", Bell System Tech. J., vol. 37, no. 2, pp. 401-446, 1958.

[33] W. C. Niehaus, T. E. Seidel, and D. E. Iglesias, "Double-drift impatt diodes near $100 \mathrm{GHz}$ ", IEEE Trans. on Elec. Dev., vol. 20, no. 9, pp. 765-771, 1973.

[34] T. Ishibashi, M. Ino, T. Makimura, and M. Ohmori, "Liquidnitrogen-cooled submillimetre-wave silicon IMPATT diodes", Electron. Lett., vol. 13, no. 10, pp. 299-300, 1977.

[35] E. J. Reijerse, "High-frequency EPR instrumentation", Appl. Magnet. Resonance, vol. 37, no. 1, pp. 795-818, 2009, (doi: 10.1007/s00723009-0070-y).

[36] R. D. Hogg, "Applications of IMPATT diodes as RF sources for microwave EPR spectroscopy", Rev. of Scien. Instruments, vol. 44, no. $5,1973$.

[37] T. Idehara et al., "Continuously frequency tunable high power sub$\mathrm{THz}$ radiation source-gyrotron FU CW VI for $600 \mathrm{MHz}$ DNP-NMR spectroscopy", J. of Infrared, Millimeter, and Terahertz Wav., vol. 31, no. 7, pp. 775-790, 2010 (doi: 10.1007/s10762-010-9643-y).

[38] V. Denysenkov, M. J. Prandolini, M. Gafurov, D. Sezer, B. Endeward, and T. F. Prisner, "Liquid state DNP using a $260 \mathrm{GHz}$ high power gyrotron", Phys. Chem. Chem. Phys., vol. 12, no. 22, pp. 5786-5790, 2010 (doi: 10.1039/C003697H).

[39] V. Flyagin, A. Gaponov, M. Petelin, and V. Yulpatov, "The gyrotron", IEEE Trans. on Microw. Theory and Techniq., vol. 25, no. 6, pp. 514-521, 1977 (doi: 10.1109/TMTT.1977.1129149).

[40] G. S. Nusinovich, P. Sprangle, C. A. Romero-Talamas, and V. L. Granatstein, "Range, resolution and power of $\mathrm{THz}$ systems for remote detection of concealed radioactive materials", J. of Appl. Phys., vol. 109, no. 8, 083303, 2011 (doi: 10.1063/1.3572062).

[41] V. Bajaj et al., "Dynamic nuclear polarization at $9 \mathrm{~T}$ using a novel $250 \mathrm{GHz}$ gyrotron microwave source", J. of Magnet. Resonance, vol. 213, no. 2, pp. 404-409, 2011 (doi: 10.1016/j.jmr.2011.09.010) [Online]. Available: http://www.sciencedirect.com/science/article/ pii/S1090780711003223

[42] T. Fujiwara, Y. Matsuki, and M. Toda, "Application of high-frequency gyrotrons to high-field DNP-NMR spectroscopy", in Proc. 5th Int. Worksh. on Far-Infrared Technol. IW-FIRT 2014, Fukui, Japan, 2014.

[43] S. Mitsudo and Y. Fujii, "Intense and short millimeter wave pulse generation by using a gyrotron as a light source", in Proc. 5th Int. Worksh. on Far-Infrared Technol. IW-FIRT 2014, Fukui, Japan, 2014.

[44] M. Hruszowiec, W. Czarczyński, E. F. Pliński, and T. Więckowski, "Gyrotron technology", J. of Telecommun. and Inform. Technol., no. 1 , pp. 68-76, 2014.

[45] A. Torrezan et al., "Continuous-wave operation of a frequencytunable $460-\mathrm{GHz}$ second-harmonic gyrotron for enhanced nuclear magnetic resonance", IEEE Trans. on Plasma Sci., vol. 38, no. 6, pp. 1150-1159, 2010 (doi: 10.1109/TPS.2010.2046617).

[46] T. Tatsukawa, T. Maeda, H. Sasai, T. Idehara, M. Mekata, T. Saito, and T. Kanemaki, "ESR spectrometer with a wide frequency range using a gyrotron as a radiation power source", Int. J. of Infrared and Millimeter Wav., vol. 16, no. 1, pp. 293-305, 1995 (doi: 10.1007/BF02085864).

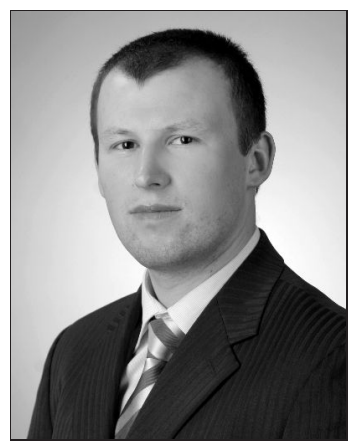

Mariusz Hruszowiec graduated from Applied Computer Science at the Wrocław University of Science and Technology in 2012. At present he is Ph.D. student at Faculty of Electronics at the Wrocław University of Technology. The main topics of his interest are gyrotron theory, electromagnetic field theory and numerical methods. 
E-mail: mariusz.hruszowiec@pwr.edu.pl

Terahertz Technology Center

Wrocław University of Science and Technology

Wybrzeże Wyspiańskiego st 27

50-370 Wrocław, Poland

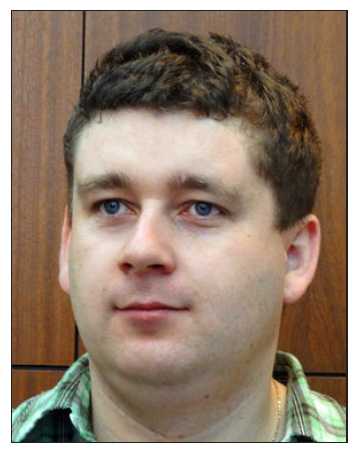

Kacper Nowak received his Ph.D. from Wrocław University of Science and Technology in 2012. He is currently an Assistant Professor in the Electronics and Telecommunication Department in the Faculty of Electronics at Wrocław University of Science and Technology. His research interests include: terahertz spectroscopy, industrial automation, networking and programming.

E-mail: kacper.nowak@pwr.edu.pl

Terahertz Technology Center

Wrocław University of Science and Technology

Wybrzeże Wyspiańskiego st 27

50-370 Wrocław, Poland

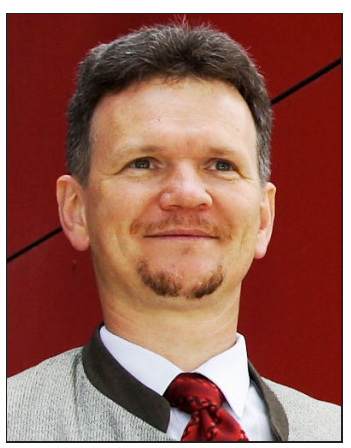

Bogusław Szlachetko received his Ph.D. from Wrocław University of Science and Technology in 2001. He is currently Assistant Professor in the Department of Signal Processing Systems in the Faculty of Electronics at Wrocław University of Science and Technology. His research interests include: digital signal processing systems, hybrid filter banks applications, multi-sensor spectral processing, and $\mathrm{THz}$ spectroscopy.

E-mail: boguslaw.szlachetko@pwr.edu.pl

Terahertz Technology Center

Wrocław University of Science and Technology

Wybrzeże Wyspiańskiego st 27

50-370 Wrocław, Poland

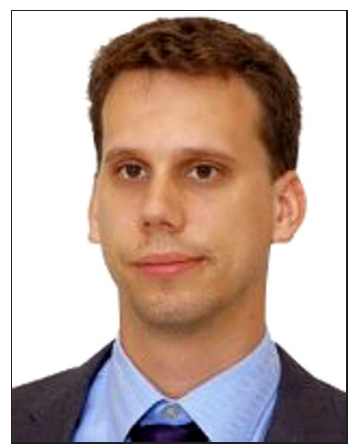

Michał P. Grzelczak is a Ph.D. student in the Department of Electronics of Wrocław University of Science and Technology. His research interests are in terahertz spectroscopy, imaging and technology itself. He received his M.Sc. in Advanced Applied Electronics from Wrocław University of Science and Technology in July 2014. He also received a B.Sc. in Electronics from Gdańsk University of Technology in February 2013. He is currently working in the Wrocław Terahertz Team towards $\mathrm{THz}$ spectroscopy.

E-mail: michal.grzelczak@pwr.edu.pl

Terahertz Technology Center

Wrocław University of Science and Technology

Wybrzeże Wyspiańskiego st 27

50-370 Wrocław, Poland

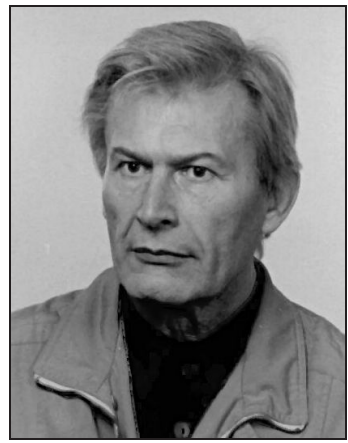

Wojciech Czarczyński received the M.Sc. in Electronics from the Wrocław University of Technology, Poland, in 1957. From 1956 to 1964 he was with the Industrial Institute of Electronics, Wrocław Branch (PIE). In the years 1964-1965 he was Research Fellow on ONZ Fellowship at the Southampton University involved in the design and research in the field of power microwave tubes. From 1965 to 1978 he was again with PIE as a head of microwave tube laboratory. In 1956 he received the Ph.D. degree in the electron beam research from the WUT. In 1978 he joined the Institute of Electron Technology, WUT, where he was involved in electron beam and plasma research. He was appointed the Institute Head for the 1987-1990 term. In 1995 he received D.Hab. degree from the Faculty of Electronics, WUT and was appointed University Professor. In 2001 he became full professor. He retired in 2003 and was a part-time research worker till 2008. Currently is the voluntary member of the Terahertz Center of the WUT, Wrocław, Poland.

E-mail:

Terahertz Technology Center

Wrocław University of Science and Technology

Wybrzeże Wyspiańskiego st 27

50-370 Wrocław, Poland

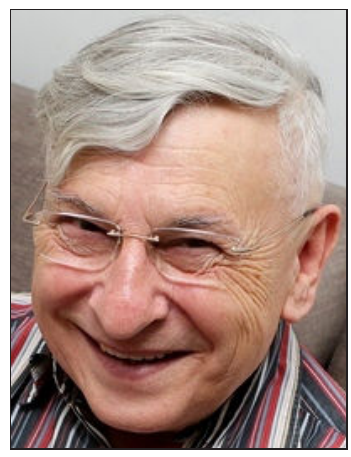

Edward F. Pliński received his M.Sc. degree in Solid State Physics from the Faculty of Mathematics, Physics, and Chemistry at Wrocław University. Since 1975 he has been with the Wrocław University of Science and Technology, where he received his Ph.D. degree in 1983. In 1985 he joined the Twente University, Enschede, in the Netherlands, where he worked with the Professor W. J. Wittemans group as a postdoctoral fellow. He received a D.Sc. in Technical Sciences in 2002. He has been a Full Professor since 2013. His primary subjects are waveguide RF excited carbon dioxide lasers. In 2006 he created the first THz group in Poland and he is a founding director of the Scientific Center of the Terahertz Technique, expanded in 2012 to include the gyrotron technique. Cur- 
rently, his subject of interest are terahertz and gyrotron techniques.

E-mail: edward.plinski@pwr.edu.pl

Terahertz Technology Center

Wrocław University of Science and Technology

Wybrzeże Wyspiańskiego st 27

50-370 Wrocław, Poland

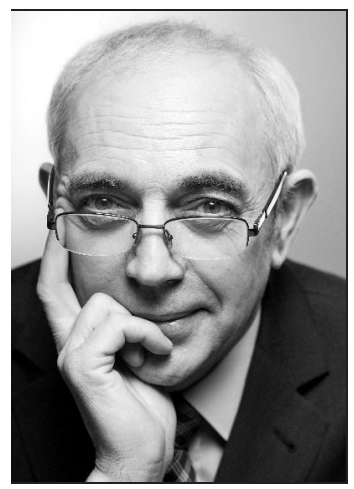

Tadeusz Więckowski specializes in the field of electromagnetic compatibility of device, systems and installations, in particular the intersystem compatibility of radio communication and telecommunication installations. He is the author of over 175 scientific publications, 6 patents and patent issues, and over 600 elaborations on economy. With the sup- port of his colleagues he initiated, created and promoted the world-class Electromagnetic Laboratory of Compatibility. One of his greatest successes is the creation of the Knowledge and Innovation Community for Information and Communication Technologies, and The Academic Incubator of Entrepreneurship at Wrocław University of Technology. For his scientific and teaching activity and cooperation with industry Professor Więckowski was twice awarded by the Prime Minister of Poland. He was honored with the Golden Badge of Wrocław University of Technology, Medal of the Commission of National Education and Silver and Gold Cross of Merit, Order of Rebirth of Poland. He is doctor honoris causa of Lviv Polytechnic National University, honorary professor of Obuda University.

E-mail: tadeusz.wieckowski@pwr.edu.pl

Terahertz Technology Center

Wrocław University of Science and Technology

Wybrzeże Wyspiańskiego st 27

50-370 Wrocław, Poland 